\title{
Manufacturing Challenges and Benefits when Scaling the HIAD Stacked-Torus Aeroshell to a 15m-Class System
}

\author{
F. McNeil Cheatwood \\ NASA Langley Research Center \\ Hampton, VA 23681 \\ 757-864-2984 \\ F.M.Cheatwood@nasa.gov
}

\author{
Gregory T. Swanson \\ AMA Incorporated \\ NASA Ames Research Center \\ Moffett Field, CA 94035 \\ 650-604-4433
}

Gregory.T.Swanson@nasa.gov

\section{Brian Gilles, Paul Anderson \\ Airborne Systems}

Lake Elsinore, CA 92530

Brian.Gilles@airborne-sys.com

Paul.Anderson@airborne-sys.com

\author{
R. Keith Johnson \\ NASA Langley Research Center \\ Hampton, VA 23681 \\ 757-864-7681 \\ R.K.Johnson@nasa.gov
}

\author{
Bruce Bond \\ Jackson-Bond Enterprises \\ Dover, NH 03820 \\ Bruce.Bond@jacksonbondllc.com
}

\begin{abstract}
Over a decade of work has been conducted in the development of NASA's Hypersonic Inflatable Aerodynamic Decelerator (HIAD) deployable aeroshell technology. This effort has included multiple ground test campaigns and flight tests culminating in the HIAD project's second generation (Gen2) aeroshell system. The HIAD project team has developed, fabricated, and tested stacked-torus inflatable structures (IS) with flexible thermal protection systems (F-TPS) ranging in diameters from 3-6m, with cone angles of 60 and 70 deg. To meet NASA and commercial near term objectives, the HIAD team must scale the current technology up to $12-15 \mathrm{~m}$ in diameter. Therefore, the HIAD project's experience in scaling the technology has reached a critical juncture. Growing from a $6 \mathrm{~m}$ to a $15 \mathrm{~m}$-class system will introduce many new structural and logistical challenges to an already complicated manufacturing process.
\end{abstract}

Although the general architecture and key aspects of the HIAD design scale well to larger vehicles, details of the technology will need to be reevaluated and possibly redesigned for use in a $15 \mathrm{~m}$ class HIAD system. These include: layout and size of the structural webbing that transfers load throughout the IS, inflatable gas barrier design, torus diameter and braid construction, internal pressure and inflation line routing, adhesives used for coating and bonding, and F-TPS gore design and seam fabrication. The logistics of fabricating and testing the IS and the F-TPS also become more challenging with increased scale. Compared to the $6 \mathrm{~m}$ aeroshell (the largest HIAD built to date), a $12 \mathrm{~m}$ aeroshell has four times the crosssectional area, and a $15 \mathrm{~m}$ one has over six times the area. This means that fabrication and test procedures will need to be reexamined to account for the sheer size and weight of the aeroshell components. This will affect a variety of steps in the manufacturing process, such as: stacking the tori during assembly, stitching the structural webbing, initial inflation of tori, and stitching of F-TPS gores. Additionally, new approaches and hardware will be required for handling and ground testing of both individual tori and the fully assembled HIADs.

There are also noteworthy benefits of scaling up the HIAD aeroshell to a $15 \mathrm{~m}$-class system. Two complications in working with handmade textile structures are the non-linearity of the material components and the role of human accuracy during fabrication. Larger, more capable, HIAD structures should see much larger operational loads, potentially bringing the structural response of the material components out of the nonlinear regime and into the preferred linear response range. Also, making the reasonable assumption that the magnitude of fabrication accuracy remains constant as the structures grow, the relative effect of fabrication errors should decrease as a percentage of the textile component size. Combined, these two effects improve the predictive capability and the uniformity of the structural response for a $12-15 \mathrm{~m}$ HIAD.

In this paper, the challenges and associated mitigation plans related to scaling up the HIAD stacked-torus aeroshell to a 15mclass system will be discussed. In addition, the benefits of enlarging the structure will be further explored.

\section{TABLE OF CONTENTS}

1. INTRODUCTION ..................................................1

2. HIAD AEROSHELL OVERVIEW .........................2

3. IS SCALING CHALLENGES.................................4

4. F-TPS SCALING CHALLENGES...........................8

6. HIAD AEROSHELl SCALING BENEFITS .......10

7. CONCLUSION AND FUTURE WORK..................11

ACKNOWLEDGEMENTS.........................................11

REFERENCES......................................................11

BIOGRAPHIES..........................................................11

\section{INTRODUCTION}

To support NASA's long term goal of landing humans on Mars, technologies which enable the interplanetary delivery of heavy payloads are being developed. Available entry, decent, and landing (EDL) technologies are not practical for Mars human-class payloads due to the geometric constraints dictated by current and anticipated launch vehicle fairing limitations. Therefore, both new and old technologies are being explored to provide a mass- and volume-efficient solution to atmospheric entry, including hypersonic inflatable aerodynamic decelerators (HIADs).

Inflatable aerodynamic decelerators (IADs) were first conceptualized at NASA LaRC in the 1960s as a viable technology for crewed atmospheric reentry. This concept was vetted by NASA and the Air Force through many wind tunnel and flight tests until the mid-1970s, when the 
technology became a candidate for the upcoming Viking, Pioneer Venus, and Galileo missions. At the same time, the disk-gap-band (DGB) parachute was reaching maturity, but had experienced issues above Mach 2, lending the advantage to IADs that had shown potential as a supersonic decelerator through testing. Unfortunately for the development of IADs, it was determined that Viking did not require performance above Mach 2 and the decelerator selection was redirected to the more familiar DGB parachute technology. As a result, the IAD technology was tabled indefinitely [1]. Recent interest in heavy payloads and different planetary destinations that require performance outside the DGB flight envelope, along with the development of new high performance textiles, has revitalized IAD technology development.

In 2006 NASA funded the Program to Advance Inflatable Decelerators for Atmospheric Entry (PAIDAE) to begin steps to mature IAD technology for present day mission infusion. This included evaluating various geometries and materials through wind tunnel tests, thermal tests, and ballistic range tests [2]. In parallel with PAIDAE, the Inflatable Reentry Vehicle Experiment (IRVE) flight test was planned and launched in 2007. IRVE was to perform a sub-orbital flight test of a $3 \mathrm{~m}$ HIAD. Unfortunately due to a launch vehicle anomaly, IRVE never got the opportunity to deploy and reenter. Given the potential of the HIAD as an enabling technology it was determined that the experiment had sufficient merit to be reattempted. In 2009 a build-to-print IRVE flight article was fabricated and launched as IRVE-2. This test was a great success, meeting all test objectives gaining it recognition as the first fully-successful HIAD flight in history [3]. With the momentum from the success of IRVE2 the HIAD team began a four-year ground-test campaign to improve the IS and F-TPS performance. The ground test campaign climaxed in wind tunnel testing of $3 \mathrm{~m}$ and $6 \mathrm{~m}$ HIAD structures [4]. In addition, high temperature materials component testing, structural component testing, and many other investigations were performed to further the technology. This work culminated in the Gen-1 IS $\left(250^{\circ} \mathrm{C}\right.$ capable) and F-TPS (30W/ $\mathrm{cm}^{2}$ capable) flown on IRVE-3, and also aided development of the Gen-2 systems $\left(400^{\circ} \mathrm{C}\right.$ and $50 \mathrm{~W} / \mathrm{cm}^{2}$ capable, respectively) that have been ground tested and are ready for mission infusion for a $5 \mathrm{~m}$ and $10 \mathrm{~m}$ class HIAD. An additional HIAD sub-orbital flight test was conducted as IRVE-3 in 2012 near the end of the ground test effort. This test improved upon the IRVE-2 flight by employing a larger sounding rocket, resulting in a higher reentry energy, and, in turn, using the improved Gen-1 IS and F-TPS to mitigate this increased energy. IRVE-3 also incorporated a center-of-gravity (CG) offset feature that allowed it to successfully demonstrate a controlled lifting entry [5]. The IRVE flight test and HIAD ground test campaigns were both fruitful activities providing high technology readiness for missions requiring $5 \mathrm{~m}$ and $10 \mathrm{~m}$ class aeroshells. These successes have propelled the technology toward further development for eventual mission use.
Opportunities are on the horizon that require larger aeroshell diameters than what can be accommodated in launch vehicle fairings using existing rigid aeroshell technology. These missions include NASA's technology development for heavy payloads on its 'Journey to Mars'. Using a flexible HIAD architecture can circumvent geometric limitations unlike traditional rigid systems that must be fixed in size and shape. As part of NASA's technology development effort, a 2024 Mars EDL pathfinder opportunity could include a $10-12 \mathrm{~m}$ HIAD as an incremental step towards the $15-20$ m class HIAD that would be required for human Mars applications. Additionally, HIAD technology offers the opportunity to return cargo from the International Space Station (ISS) through integration with the current fleet of resupply vehicles. These cargo ships rendezvous with the ISS to deliver their payloads, then are packed with trash and released for disposal, ultimately disintegrating in the Earth's atmosphere. A $10 \mathrm{~m}$ HIAD would enable a down-mass system to safely return payloads to Earth, creating more opportunities for the scientific community interested in retrieving science payloads. Finally, the commercial launch provider, United Launch Alliance (ULA), recently expressed interest in HIAD technology to enable the reuse of their firststage engines. ULA has announced that they will use a HIAD (presumably $12 \mathrm{~m}$ ) as part of their Sensible, Modular, and Autonomous Return Technology (SMART) for their upcoming Vulcan rocket. ULA claims that the SMART reusability initiative, shown in Figure 1, will reduce their booster cost by $65 \%$, sustainably collapsing the cost of upmass capability making ULA more competitive within the commercial sector. This initiative is targeted to begin operation in 2024 [6].

Each of these opportunities require HIAD systems larger than what has been manufactured and tested to date. This will require the HIAD project team to explore scalability of the current technology and generate a development plan to meet the need for larger aeroshells. This effort will introduce many manufacturing challenges not experienced by the project team or vendors during prior fabrication.

\section{HIAD Aeroshell OVERVIEW}

The HIAD aeroshell is comprised of two main components, the inflatable structure (IS) and the flexible thermal protection system (F-TPS). The IS design defines the geometry of the HIAD and provides the strength to withstand the aerodynamic pressure loads seen on reentry. The F-TPS is designed to shield the IS and the mission's payload from the aerothermal heating experienced during atmospheric entry. The current IS is fabricated by Airborne Systems in Lake Elsinore, CA, and the F-TPS is fabricated by Jackson Bond Enterprises in Dover, NH. 


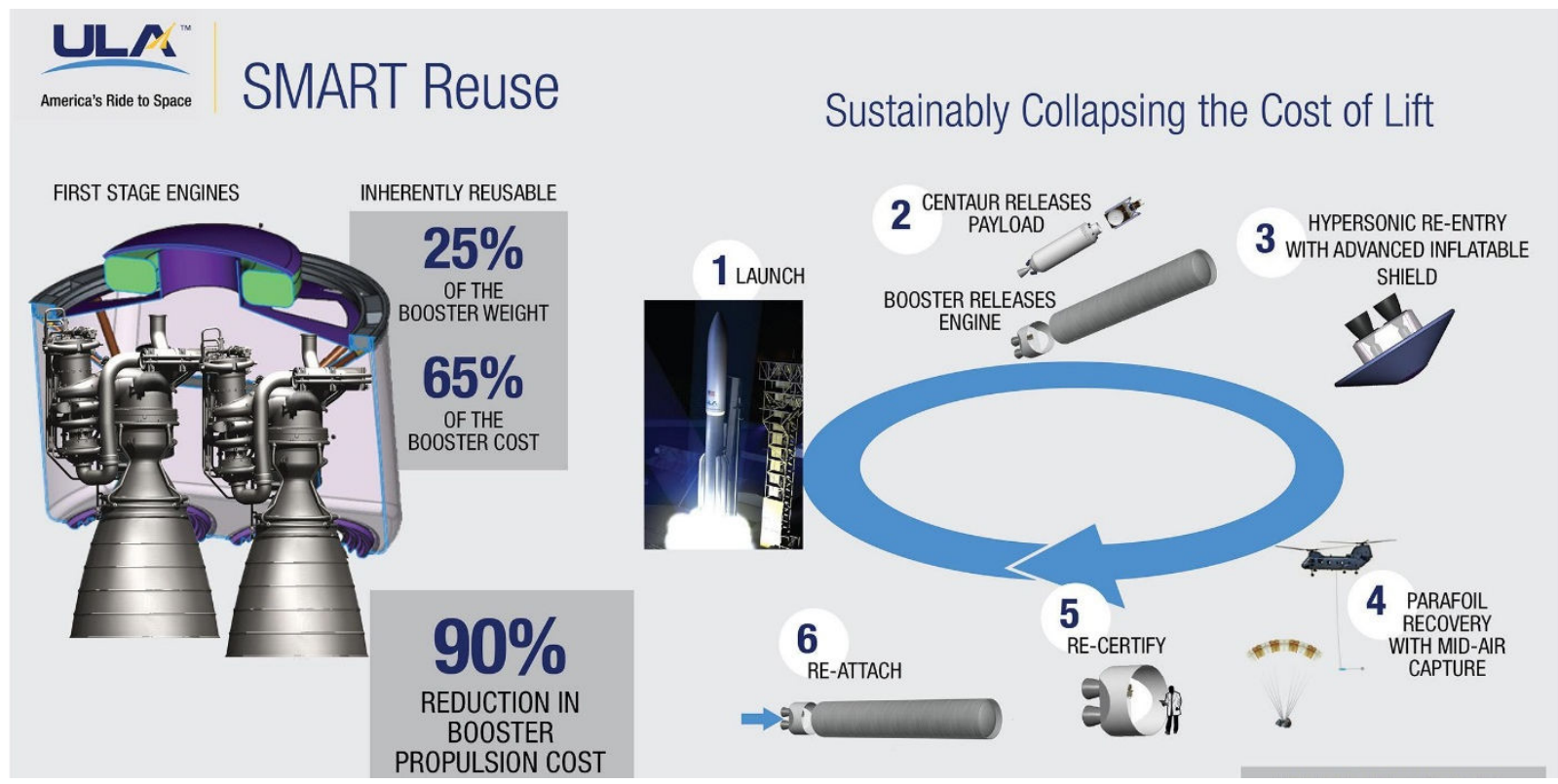

Figure 1 - ULA SMART Reuse Overview [6]

\section{Inflatable Structure Design Overview}

The Gen-1 and Gen-2 HIAD IS design consists of stacked inflatable torus segments that are joined together by structural fabric straps (or webbing). This structure is then anchored to a rigid centerbody with additional webbing. A crosssectional view of the $3.7 \mathrm{~m}$ HIAD IS design is shown in Figure 2, and the fabricated structure is shown in Figure 3. A liner material on the inside of each torus acts as an inflatable bladder. On the outside of the torus a textile braid is employed to protect the liner and give strength to the system. These inflatable tori are sized to meet the required HIAD cone-angle and cross-sectional area. To distribute the pressure load experienced during reentry throughout the stack of inflatable tori, load-bearing straps are integrated with the structure. As shown in Figure 4, these designs can be broken into three categories: pairing straps, radial and chevron straps, and centerbody attachment straps.

- Pairing straps wrap around two adjacent tori and secure them together. These straps carry load on both the forward and aft sides of the article. Pairing straps are patterned evenly throughout the structure in staggered groups.

- Radial straps teamed with chevron straps (also known as "crow's feet") are designed to transfer load from the outer tori directly to the centerbody to alleviate the inward-cascading load that is seen in the paring straps throughout the structure. These straps are anchored at the rigid centerbody, then run outward and wrap around outer tori, effectively bypassing the inward-cascading load path. These radial/chevron straps are evenly distributed around the IS and can be used at multiple outboard positions depending on the design requirements.
- Centerbody attachment straps connect the smallest (inner most) torus (T1) to the rigid centerbody. Theses straps are anchored to clevis pins on the forward and aft side of the centerbody.

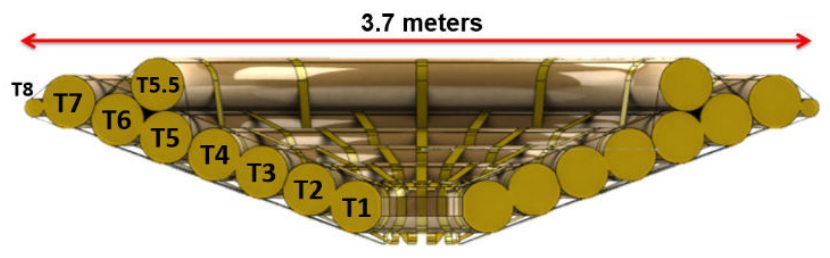

Figure 2 - Cross-Sectional View of the 3.7m HIAD IS

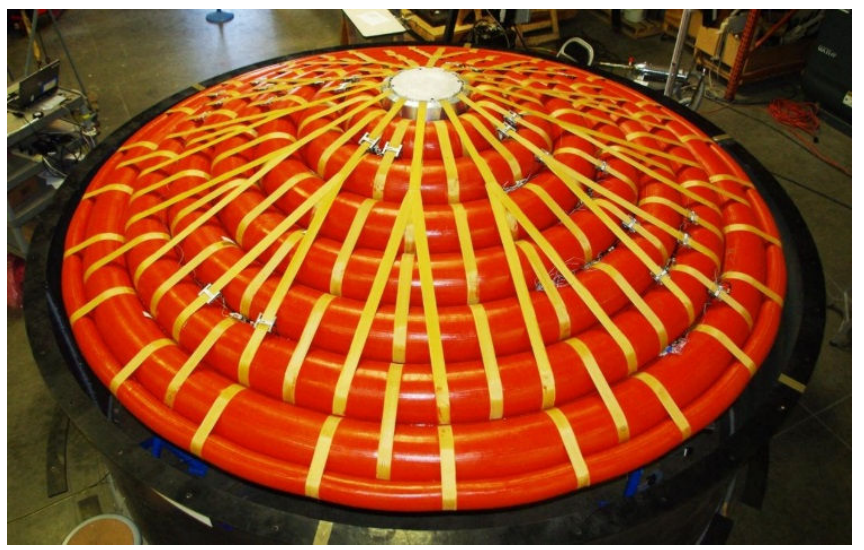

Figure 3 - 3.7m Inflatable Structure Fabricated in 2014 


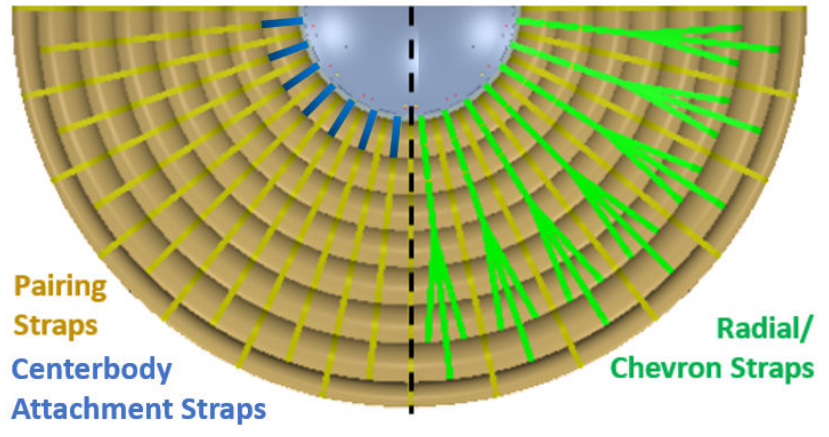

Figure 4 - Pairing Straps (left), Centerbody Attachment Straps (left), and Radial/Chevron Straps (right)

\section{F-TPS Design Overview}

The F-TPS consists of multiple layers of material to provide thermal protection to the structure, as seen in Figure 5. These layers are stitched together in specialized patterns to lock the position of the materials, provide strength to the system, and mitigate wrinkling and bulging of the outer material that can cause aerothermal complications, all while maintaining flexibility of the system. The F-TPS is anchored at the rigid centerbody using radial webbing, and terminates around the shoulder of the IS on the aft side of the HIAD. The multiple layers used in the F-TPS can be broken into three categories: outer fabrics, insulators, and gas barrier.

- The outer fabrics mitigate the initial aerothermal heating and the shearing force from the flow experienced during entry. The F-TPS design commonly employs two layers of outer fabric. Depending on the mission, different materials can be used to meet performance requirements of the entry trajectory.

- The insulators decrease the temperature to keep the gas barrier and tori below their maximum operating temperatures. The number of insulator layers included in the F-TPS varies depending on the anticipated total heat load for the mission.

- The gas barrier is designed to prevent the hot gasses experienced during atmospheric entry from passing through the system to the IS. The gas barrier also provides a semi-rigid structure on which to anchor the multiple F-TPS layers through the specialized stitching designs.

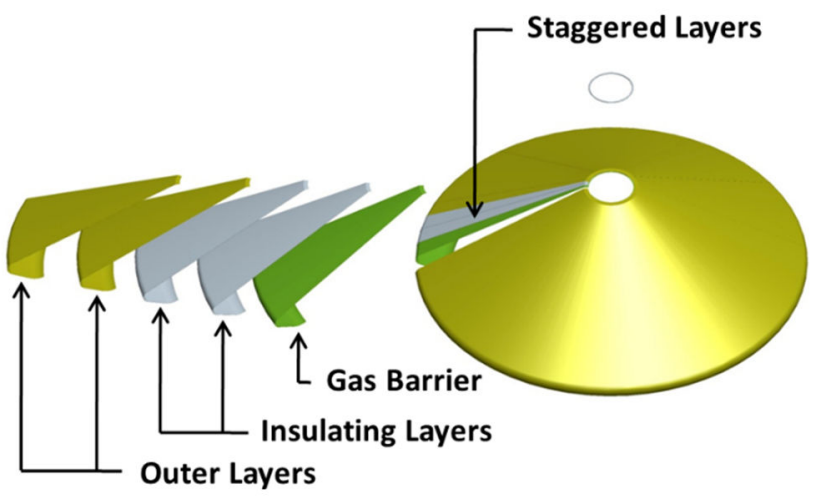

Figure 5 - F-TPS Layout Drawing

\section{IS SCALING ChallengeS}

The HIAD technology has reached a critical juncture in development, as the architecture must now be scaled to a $15 \mathrm{~m}$-class system to meet near and long term goals of NASA and commercial spaceflight. Although the general architecture and key aspects of the design scale well to larger vehicles, details of the technology need to be reevaluated and possibly redesigned for use in a $12 \mathrm{~m}-15 \mathrm{~m}$ HIAD system. A size comparison between the aeroshell structures that have been built to date, and a $12 \mathrm{~m}$ IS can be seen in Figure 6, displaying the leap in scale. The HIAD team is developing a plan of action to address the associated challenges over the next couple of years, culminating in the fabrication and ground testing of a $12 \mathrm{~m}$ aeroshell.

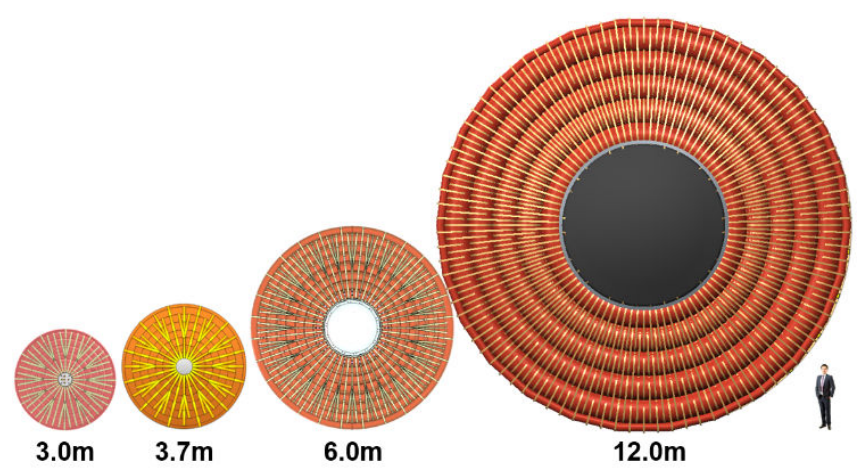

Figure 6 - HIAD IS Built to Date (3m, 3.7m, and $6 \mathrm{~m})$ vs. $12 \mathrm{~m}$ IS, with $6 \mathrm{ft}$ Person for Reference

\section{Structural Strap Layout}

As described in section 2, the HIAD structure is bound together using high strength webbing. The current $6 \mathrm{~m}$ system has 28 strap positions evenly distributed across the inflatable article as shown in Figure 4. Pairing straps bind together adjacent tori while the radial/chevron straps wrap around the outer tori to shunt the cascading load path back to the rigid centerbody. These straps currently range from 1.75 " to 2 " in width. This layout has been proven to withstand $50,000 \mathrm{lb}$ of load when integrated with the F-TPS at IS internal pressures down to 10 psi. Scaling this system to a $15 \mathrm{~m}$-class will require a redesign of the strap layout, trading the width and 
strength of the individual straps with the number of strap positions to provide better coverage and higher global load capability. To address this challenge, a series of componentlevel tests will be conducted to determine the performance of different strap designs.

One of the main performance factors evaluated for the structural strap design is the indentation of a strap as it travels around a torus at a given internal inflation pressure. If the load in the strap increases to the point that the force overcomes the structural integrity of the torus, severe indentation and a subsequent loss of load-carrying capability in the strap can occur, causing a redistribution of the load to neighboring straps. To mitigate indentation at the higher loads that will be seen during $15 \mathrm{~m}$-class missions, wider straps providing a greater surface area to distribute load across the tori will be tested. However, increasing the strap width raises a concern about the potential for edge loading in the strap, which can cause localized stress. On a straight beam this could cause complications, but given the inherent curvature of the tori, the edge loading might actually provide a form fitting interface.

Another concept for mitigating strap indentation marries the current 1.75 " or 2 " webbing with a textile 'basket'. The basket would be three to four times wider than the original webbing and would cradle the torus, providing a greater surface area to distribute the load. Subscale component tests will be done to evaluate this approach. Once the optimal strap design/system is determined, analyses will be conducted using the computational tools developed by the HIAD team to define strap layout. As the system grows from a $6 \mathrm{~m}$ to a $15 \mathrm{~m}$-class structure, the aeroshell will likely require additional strap positions to regulate loads carried by each strap. In addition to the number of strap positions, the number of outboard chevrons will also be evaluated. With the great increase in size of the $15 \mathrm{~m}$ system, it may be determined that two sets chevron straps are required at each position. For example, one set could terminate at T3 and another at $\mathrm{T} 6$ along the same radial position.

\section{Inflatable Gas Barrier Design}

As described previously, each torus of the stacked-torus HIAD design includes a high elongation, low permeability liner material that acts as the inflation gas barrier. The inflatable liner is currently fabricated by trimming material to the required size, then using an adhesive bond to form an axial seam, creating a cylindrical structure. This tube is then inserted into the structural braid and the ends are sealed together creating the torus. In an effort to provide the greatest structural efficiency, the minor diameter of the tori will increase as the scale of the HIAD aeroshell increases. The Gen-2 liner material manufacturer currently has a maximum width of 54". Wider processes could be developed, but at too great an expense for the current project. The 54" limitation has not been an issue to date since the largest minor diameter fabricated has been 13.6" (on the 6m HIAD), which required a liner material width of approximately 43 ".
To make tori with larger minor diameters the HIAD team plans to use multiple axial seams to circumvent the asmanufactured liner width limitation. As described above, the current fabrication process for the gas barrier involves creating a straight tube that is then formed into a torus. This results in more strain on the outer side of the liner as compared to the inner side. The current one-seam liners are positioned so the axial seam is on the inner-most point of the torus where it will experience the least strain. As the seam moves away from that inner-most point, it will see increasing strain due to the curvature of the torus. This presents itself as a challenge for larger tori that require liners with multiple seams. For instance, fabricating a 32" minor diameter torus with the manufacturer's 54" liner width constraint would require two axial seams. If one seam is kept at the inner-most point for minimum strain, the other seam would be located at the outer-most point and would see the worst-case strain. However, if the seams are positioned at approximately the top and bottom of the torus they will share the strain equally, which avoids exposing either to the worst case strain. From a thermal standpoint, the worst case strain design specified above is advantageous since each seam would be located in the interference area (between tori) of the stacked torus structure, which would provide additional protection for the seams. These tradeoffs must be evaluated moving forward. Going larger than a 32" minor diameter torus would require the introduction of a third axial seam, adding greater complexity to the structure. Initial fabrication of a two-seam torus has been conducted and successfully tested by the HIAD team, providing merit to the concept. To further evaluate the multiple liner seam approach, additional lowcost subscale articles will be created and tested in different configurations. This will provide the insight required before the team defines the design of larger minor diameter tori for a $15 \mathrm{~m}$-class HIAD system.

\section{Torus Braid Design}

As described in section 2, each torus of the stacked-torus HIAD design includes a high strength braided tube that protects the gas barrier and provides strength to the structure. The largest HIAD braid that has been fabricated to date was for the construction of the $6 \mathrm{~m}$ article. This braid was approximately $13.6 "(0.35 \mathrm{~m})$ in diameter. But once again, as the scale of the HIAD aeroshell increases, so will the minor diameter of the tori, and thus the braid itself. Scaling of the braid can be accomplished within the woven design variables and manufacturer limitations. The vendor working with the HIAD project (A\&P Technology) can braid up to a 24 " cylinder of the current design on their 800-carrier machine. Given the number of carriers, denier of fibers, and braid angle used for the HIAD tori, the braid for a cylinder larger than 24" would exhibit gaps (or windowing) between the braided yarns. This windowing behavior could allow the gas liner to protrude through the braid, leaving it susceptible to damage. The fibers per carrier bundle could be increased to create greater coverage, but this would be an inefficient solution due to increased braid thickness and mass. Alternatively, the braid angle can be modified to get the desired coverage at larger diameters, but this changes the load carrying capability 
of the braid. Additional carriers may be added to the machine to achieve increased coverage mitigating windowing for diameters greater than 24", but this will require an additional investment. The HIAD team is still evaluating these options.

Another option to get the desired coverage while maintaining or reducing the number of carriers is to braid with ribbons. This could provide much more surface area for coverage compared to the traditional fiber yarns without a noticeable reduction in the strength of the structure. Braiding with ribbons has already been demonstrated by HIAD vendors and initial prototyping of torus structures will be performed and tested to evaluate the viability of the alternative ribbon structure. Once this data has been collected and analyzed, the team will decided whether the investment to add carriers to the A\&P braider is necessary.

\section{Material Availability and Quality}

The evolution of high performance materials has enabled the development of IADs for future missions. These materials have often been invented for specific applications, which the HIAD team must repurpose for integration into the inflatable aeroshell. Others materials, such as the textiles, come in the raw fiber form and must be fabricated into the desired braid and webbing. Due to the specialized nature of the current Gen-2 IS materials, most are limited in availability, resulting in long lead times for procurement. This will become a growing challenge as the IS is scaled to a $15 \mathrm{~m}$-class that requires a significantly greater quantity of materials. To alleviate this constraint, alternate materials with similar properties and performance are being explored as candidates for IS manufacturing. Having multiple material options available for a given system will also provide technology robustness to material supply chain factors that are out of the project's control.

In addition to availability of these high performance materials, their quality can be a challenge. The HIAD structure requires large sections of defect-free material to construct the inflatable articles. Depending upon the original intended application for the material, quality or consistency may not have been a great concern. This has not been much of an issue in the past working with $6 \mathrm{~m}$ structures and smaller, but as the project scales to larger systems, this will become a greater challenge. For example, a $6 \mathrm{~m}$ torus requires approximately $19 \mathrm{~m}(\sim 62 \mathrm{ft})$ of defect-free material, whereas a $15 \mathrm{~m}$ torus will require approximately $45 \mathrm{~m}(\sim 148 \mathrm{ft})$. This is more than double the required length of pristine material for the $6 \mathrm{~m}$ torus. To alleviate this challenge the HIAD team must work with the material manufacturers to convey the quality requirements of the structure and determine ways to assure minimal defects are present in the materials. Initial discussions have taken place and manufacturers appear willing to work with NASA to improve process and product controls which helps to meet the high quality material softgoods requirements for the HIAD application.

Once the materials are received by the project, quality control inspection processes must also be put in place by the IS fabrication team to quickly identify defects before time is lost working with inferior materials. This process will use light tables with rollers and other tools to search for defects and inconsistencies. The combination of these strategies for quality assurance of materials will help the team as they move forward to $15 \mathrm{~m}$-class systems.

\section{Inflation Port and Line Design}

The current interface between the inflation system and the IS consists of flexible hoses (or lines) that mate with ports on each torus. The number of ports and lines per torus can vary given the volume and required fill time. These ports and lines must be robust enough to allow packing and deployment of the system along with the temperature extremes experienced in flight. The IRVE-3 flight system used silicone lines and aluminum ports as the inflation interface, but this was a solution for fairly modest temperatures. The current Gen-2 HIAD design uses 5/8" outer diameter (OD) silicone ports mated with Teflon lay-flat hoses, providing a higher temperature solution. The $5 / 8$ " OD port size is considered to be the largest port diameter possible given the current design without causing windowing issues that could damage the liner, and scissoring effects that could cut the port. Given this port size constraint, moving to much larger tori could be a challenge depending upon the required inflation time. Inflation time, inflation rate, and torus pressure requirements define the trade-space for the number of ports per torus. As more lines and ports are added, the fill rate will increase, but so will the mass and complexity. Mission requirements can also limit the available inflation time. For example, a HIAD system delivering a large payload to Mars may have days to inflate before entering the atmosphere, while the ULA SMART initiative expects to only have the order of 5 minutes to achieve the full inflation pressure before the system must be ready for reentry. This brief inflation time would require many more inflation ports per torus, resulting in significant additional mass and complexity of line runs. These factors may drive the HIAD team to look at new inflation port and line designs such as torus-to-torus ports. These could be fabricated using two mating ports that lie in the valley between two tori on the aft side of the structure, or even integrated ports that feed-thru the interference between the two tori. These concepts will be developed and tested to offer alternative approaches to the current 5/8" OD port limitation.

\section{Inflatable Structure Assembly}

As the HIAD aeroshell is scaled up to a $15 \mathrm{~m}$-class system, many of the current IS fabrication and assembly processes must be reevaluated. Tasks as simple as handling individual tori will become much more challenging due to the increased size and weight. Procedures and support hardware will be developed to ensure that materials are handled with the proper support to mitigate these concerns. In addition, the sheer size of these articles will cause new challenges. Just moving structures from one high-bay in the fabrication facility to another will require deflation, folding, and reinflation of the articles. It might also require the use of an overhead crane, which is not presently available at the 
manufacturer's facility. Once all the tori have been fabricated they are placed in stacking fixture to help set a consistent cone angle and spacing as shown in Figure 7. This process traditionally entails moving the tori into position by hand to set the correct interferences. At the $15 \mathrm{~m}$ scale it is likely that this manual approach will no longer be viable given the tori size. Custom adjustable tools utilizing webbing wraps to anchor the tori to the stacking fixture have been conceptualized to address this challenge.

Another challenge associated with scaling up the aeroshell is the stitching of the structural straps. Currently, after the IS and pairing straps are initially positioned, the entire structure is deflated and carried over to a sewing machine where the webbing ends are stitched together to close out the pairing strap. This was a simple process for the $3 \mathrm{~m}$ structures, but was much more challenging with the $6 \mathrm{~m}$ articles, and there is little expectation that this process will work with $15 \mathrm{~m}$-class hardware. To address this challenge the HIAD team plans to employ a highly-mobile programmable modern sewing machine so the straps can be stitched with the article still in the stacking fixture. Requests for information are being submitted to the textile industry to determine the best technology for our given application.

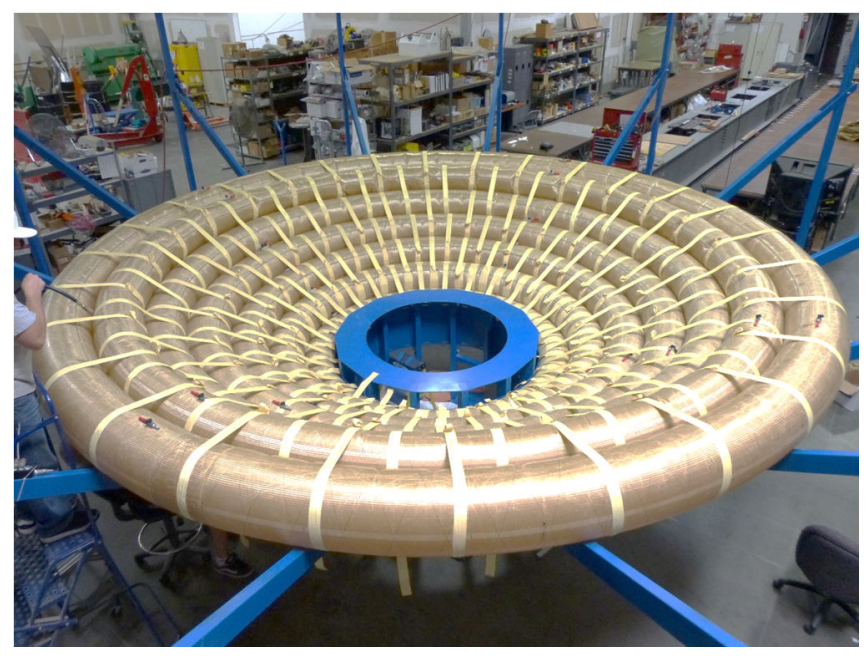

Figure 7 - Stacking Fixture for the 6m IS

\section{Inflatable Structure Component and System Testing}

The HIAD team is always looking for new ideas to increase the technology's performance envelope. When prototypes of new IS concepts or materials are constructed, a set of evaluation tests are completed to compare their performance to the baseline system. Many of these tests can be completed at sub-scale and component level, and therefore will not require any alterations to their test methods. However some tests will require additional efforts as the scale of the HIAD articles grow. After a new or altered torus construction has been prototyped (usually with a T3 or T5 torus), hydrostatic and strap indentation tests are performed. Hydrostatic testing allows the team to proof-test the torus to very high internal pressures ( $\sim 6 \mathrm{x}$ mean operating pressure) without the concern of violent, high-energy failures. Traditionally the torus is deflated, submerged in a small $4 \mathrm{~m}$ diameter pool, and then filled with water until the torus reaches the desired proof pressure. A T3 torus on the baseline $12 \mathrm{~m}$ structure will be over $8.5 \mathrm{~m}$ in diameter and therefore requires a much larger test pool and equipment. Hydrostatic testing at this scale will have to be completed at an alternate facility, unless it is determined that subscale article testing is suitable for evaluation.

Strap indentation testing entails positioning an individual torus so that weights can be hung from the strap locations to mimic the loading seen when integrated with the complete structure. Since the indentation is dependent upon the minor diameter of the torus, thickness of the structural braid, and the strap width, the test must be conducted at full scale. It would be feasible to scale all of the materials to perform a subscale indentation test, but this would require custom materials just for this purpose and would not be an efficient use of resources. The stand currently used to support indentation testing is shown in Figure 8, but new test methods to safely apply much larger loads, and equipment to accommodate the tori of a $15 \mathrm{~m}$-class HIAD will be required.

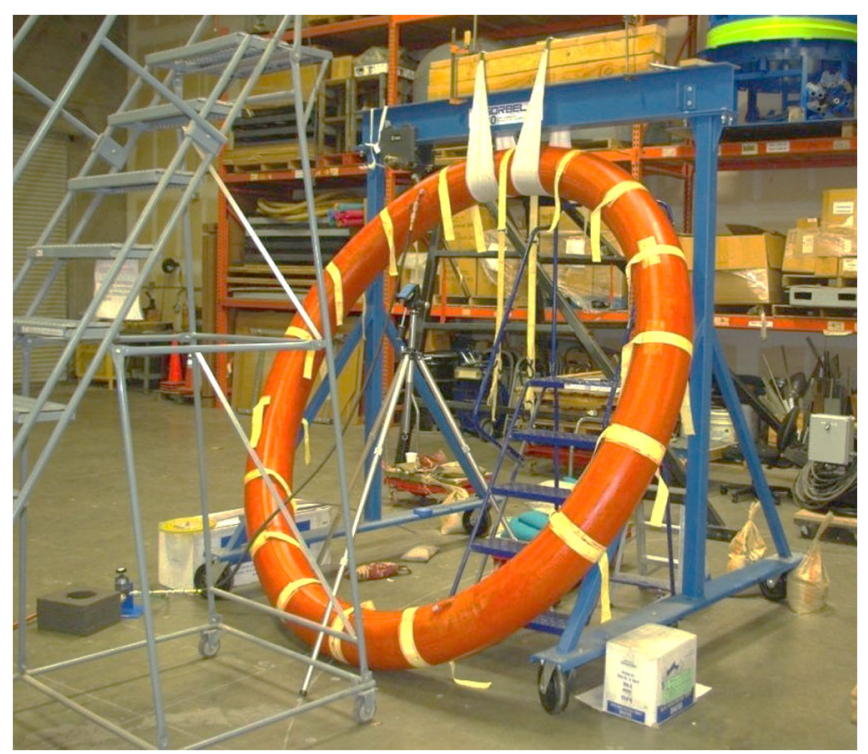

Figure 8 - Strap Indentation Test Setup Example

Once the IS fabrication is complete, the system must undergo static load testing to verify structural performance. In order to apply a static pressure distribution over the forward surface of the inflatable structure, the entire HIAD assembly is mounted in a test fixture that allows a partial vacuum to be drawn on the aft side of the article. To do so, the forward surface of the structure is covered with a nonporous transparent membrane that maintains an air-tight seal, providing an evenly distributed differential pressure between the forward and aft sides of the inflatable structure. The centerbody is supported by a hydraulic ram that reacts the pressure load, as shown in Figure 9. With the exception of the hydraulic ram, which has been used in all static load tests to date, the test fixtures are tailored to the specific size of the HIAD being tested. The current ram has a load rating of 
$50,000 \mathrm{lbs}$ and will be insufficient as we expand HIADs beyond $6 \mathrm{~m}$. The ram will need to be upgraded, and its support structure redesigned to avoid putting point loads into the concrete floor, as test loads on the order of $300,000 \mathrm{lbs}$ are expected for a $15 \mathrm{~m}$-class system.

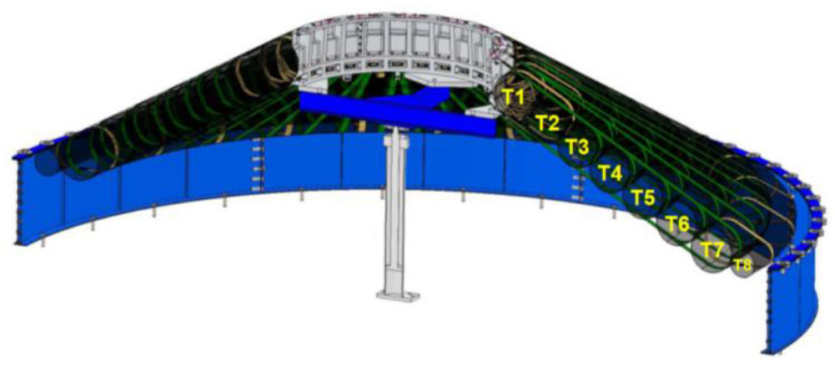

Figure 9 - Static Load Test Fixture Cross-Section

\section{F-TPS SCALING CHALLENGES}

Building on flight and wind tunnel tests, the F-TPS was advanced through incremental developments for improved thermal capabilities and increased diameters under the HIAD project. A $6 \mathrm{~m}$ diameter F-TPS was constructed and integrated with the $6 \mathrm{~m}$ IS and centerbody representing a flight vehicle interface, as shown in Figure 10. Materials and designs for the $6 \mathrm{~m}$ F-TPS were required to meet a peak heat flux of $30 \mathrm{~W} / \mathrm{cm}^{2}$ and were designated as "Gen-1". To extend F-TPS thermal performance, materials with higher temperature capabilities were developed and tested at the component level to meet a $50 \mathrm{~W} / \mathrm{cm}^{2}$ heat flux and are designated as "Gen-2". A 3.7m diameter F-TPS, shown in Figure 11, was fabricated with the Gen-2 materials to demonstrate improved construction methods.

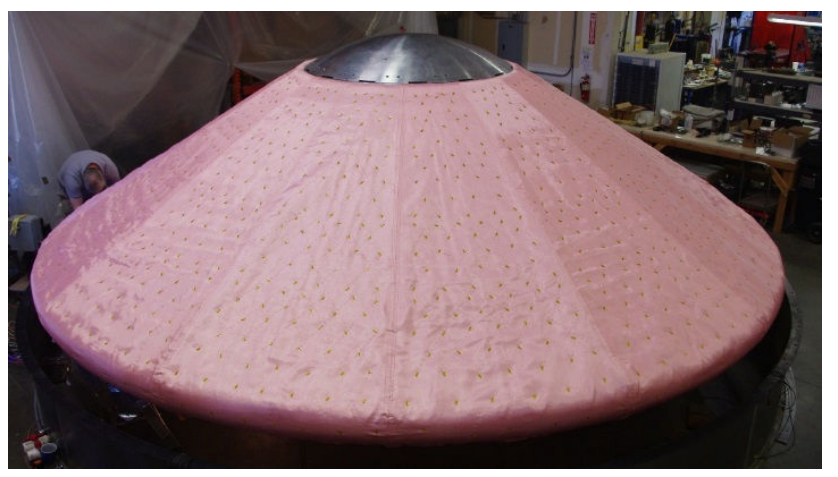

Figure 10 - 6m Diameter F-TPS (Gen-1) Integrated with IS and Centerbody

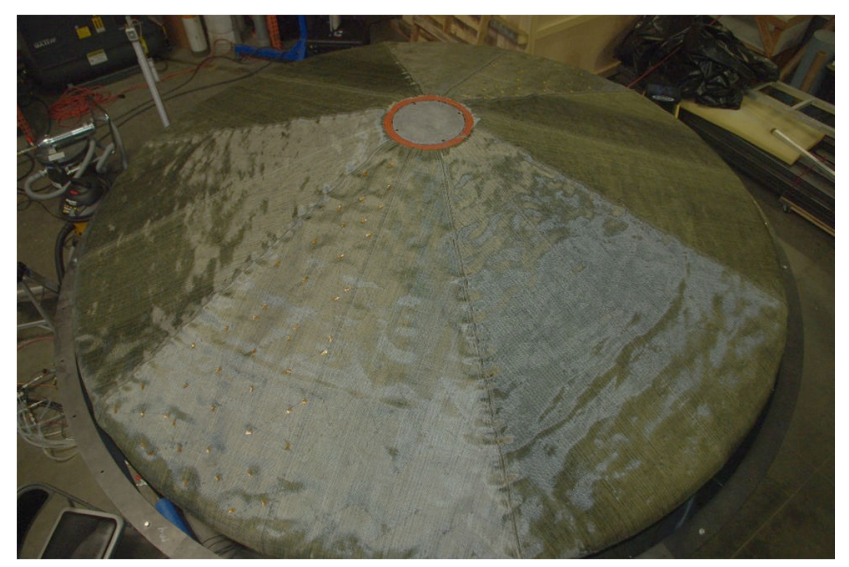

Figure 11 - 3.7m Diameter F-TPS (Gen-2) Integrated with IS and Centerbody

Increasing the size of the article from $6 \mathrm{~m}$ to $15 \mathrm{~m}$ presents many new challenges for F-TPS fabrication, handling, integration, and testing. The $6 \mathrm{~m}$ F-TPS was mostly fabricated with manual handling and lifting within a relatively small facility. A $15 \mathrm{~m}$ F-TPS will require special fixtures for handling, custom sewing equipment, and a very large clean, environmentally-controlled facility. Extending the current gore section construction and assembly methods to larger sizes presents new challenges given width limitations of available materials. The primary scaling challenges and mitigation approaches planned for $15 \mathrm{~m}$-class F-TPS are described below.

\section{Gore seaming and stitching}

As discussed in Section 2, the F-TPS is comprised of material layers specifically selected to suit the thermal environment, flight aerodynamic conditions, structural loads, and interface requirements. The F-TPS layers are designated as: outer layers for the highest temperature requirements, insulating layers tailored to meet specific temperature constraints for optimum performance of the IS, and a gas barrier layer to prevent hot gases from reaching the inflatable structure. This construction is shown in Figure 5. Each layer is made with gore sections joined with unique seams, thread and stitch designs. The sewing and stitching for the previous F-TPS articles was performed by hand or using traditional industrial sewing machines. Due to the large size and bulk of the materials for a $15 \mathrm{~m}$ F-TPS, it is expected that the sewing machines will need to be made portable and taken to the material on layout tables for sewing. Custom-designed sewing machines with drive units to synchronize movement with sewing may be required and will be explored.

The mitigation approach will begin with an investigation of sewing methods used for very large fabric structures such as for airship fabrication. Discussions will be held with companies that specialize in design and production of industrial sewing equipment and companies in the textile industry. The unique fabrics, insulating materials, and threads used for the F-TPS will need to be considered for sewing requirements and equipment capabilities. The approach to demonstrate sewing methods for our materials 
will depend on the existence of suitable equipment, or the need to fabricate custom equipment. The objective would be to demonstrate the method(s) with F-TPS material samples to reduce the risk for unexpected delays and costs during fabrication of the initial $15 \mathrm{~m}$-class F-TPS system.

\section{Limitations on Material Widths}

The gore sizes used for the $6 \mathrm{~m}$ F-TPS design required materials in widths that are currently available from the manufacturers. Insulating materials for the Gen-2 design are available up to 48 " wide. The outer layer fabric can be woven by the current manufacturer in widths up to 72". Extending the current F-TPS gore and layering design for $15 \mathrm{~m}$-class would require larger material widths than those currently available. One option to circumvent this limitation would be to increase the number of gore sections to suit the available material widths, but this comes at the cost of added complexity, increasing the number of seams, the amount of stitching required, and handling of the materials. This will increase fabrication costs and may stiffen the F-TPS, reducing its ability to meet packing requirements.

To mitigate complications related to the width limitations of available F-TPS materials, the team will explore increasing production capabilities for greater widths, identifying alternate TPS layering materials, and varying gore designs. For the woven cloth outer layers, weaving companies will be queried for equipment that can meet the enlarged size requirements for a $15 \mathrm{~m}$-class system. If significant investments in production equipment are required for greater widths of these unique high performance materials, changes to the current F-TPS gore design and material layout will also be investigated. Varying the number of gore sections and the layout of materials given fabric weave skewing and seaming will be evaluated. Trades between material width and gore sections for cost, fabrication, and packing ability will be conducted.

\section{Low-outgassing Adhesive}

Adhesives used for the previous F-TPS development, as with the IS, were not required to meet space flight low-outgassing requirements since they were stand-alone development tests. For previously fabricated F-TPS articles, the use of adhesives has been very limited, employed only for the F-TPS seam terminations at the centerbody.

Several manufacturers offer low-outgassing adhesives that comply with ASTM E595 and have been used by NASA and ESA for space vehicles. An initial survey has been conducted of available adhesives including one-part and two-part silicone adhesives that appear to be candidates for HIAD use. To determine acceptable performance with F-TPS materials and mitigate risks for future designs, several of these adhesives will be tested using subscale and component fabrication. The results will then be analyzed to determine the best low-outgassing adhesive for future use.

\section{Fabrication Equipment and Fixtures}

The $6 \mathrm{~m}$ and $3 \mathrm{~m}$ F-TPS were fabricated using relatively small tables and cutting equipment. The largest material sections were about $5 \mathrm{ft}$ wide by $10 \mathrm{ft}$ long and could be processed with existing equipment at the manufacturer's facility. For a $15 \mathrm{~m}$ F-TPS, the required material sections may be up to twice the width and three times the length. This will require larger layout tables and wider cutters, which are available commercially for purchase. The most significant equipment upgrade needed will be the much larger assembly fixture that is used to build-up the layers of the complete F-TPS. This fixture will require a very large facility space, and a custom design to suit the system's size and shape. The assembly fixture and operations will need to have provisions for personnel access to be near and above the F-TPS fixture for fabrication. Special man-rated lifting fixtures and sewing assemblies may be required to meet these operations. These are not particularly difficult technical challenges, but the cost for special equipment and fixtures needs to be considered and included in project plans.

\section{Material Lifting and Handling}

The significantly longer, wider, and heavier sections of cloth and material layers for a $15 \mathrm{~m}$ F-TPS will require special lifting and handling capabilities during fabrication. Currently most of these materials can be lifted by hand without generating large point loads. As the F-TPS grows in size, the increasing material weights will require evenly distributed lifting loads over a broad area to avoid damaging the material. Lighter single layer material sections can be rolled up for moving from the cutting and preparation area to the assembled area, but the larger and heavier sections may require special lifting fixtures or slings. For moving the assembled F-TPS, a special sling will be needed to distribute the load. The weight of a $15 \mathrm{~m} \mathrm{F-TPS}$ is expected to be $1,000-$ $2,000 \mathrm{lbs}$ and will require an overhead crane for lifting. Additional fixtures may be required for placing the heatshield over the IS for integration. Each of the lifting operations will need to be engineered to determine the lifting techniques and special equipment required. Some lifting equipment will need to be demonstrated with surrogate materials to avoid risks in damaging expensive flight-materials and articles.

\section{Facility Space Requirements}

Fabrication of a $15 \mathrm{~m}$ diameter F-TPS will require a very large facility for assembly support equipment. The facility currently used by the F-TPS contractor can accommodate diameters up to $12 \mathrm{~m}$. Larger systems will require the fabrication operations to be relocated to a larger facility, with overhead lifting capabilities, and a ceiling height of at least $25 \mathrm{ft}$. Figure 12 shows a $12 \mathrm{~m}$ assembly in the current facility's high bay area. The article is tilted to provide better access to the F-TPS shoulder area for personnel on the floor and a more level surface on the upper side for placement of material gore section layers. An assembly form representing the inflatable structure surface is used to support the F-TPS layers during assembly and has a center shaft with bearings to allow rotation, along with an adjustable hinge to change 
pitch angle. Figure 12 also emphasizes the challenges for personnel and equipment access for assembly and safe operations. Man lifts or custom scaffolding will be used to safely position fabricators over the article. Portable sewing equipment used for some gore seams must be able to be maneuvered into position and controlled by the operator. There are facilities with sufficiently large open spaces, support capabilities, and environmental controls to alleviate the current limitations, but their location and availability are very limited. Appropriate facilities will need be to investigated and evaluated for overall suitability and cost. Costs for contractor relocation and required support equipment will also need to be considered for large scale HIAD projects.

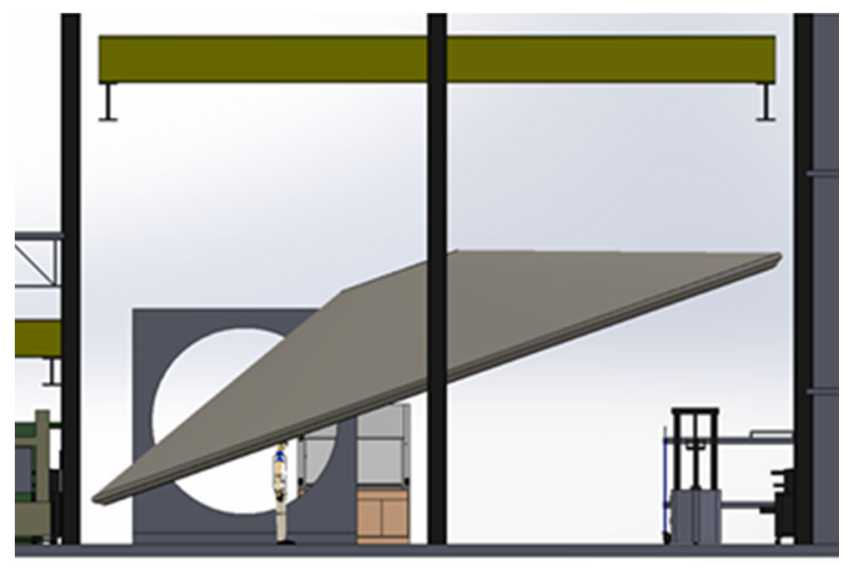

\section{Figure 12 - Current Manufacturing Facility Space with 12m F-TPS Assembly Fixture}

\section{Materials Testing, Inspections and Quality Control}

As F-TPS sizes increase, materials testing and inspections become more challenging and critical. The larger quantity of material in the form of woven cloths, insulating layers, gas barriers, webbing, and sewing thread must be verified and inspected for defects. Each material lot must be tested to ensure compliance with construction and property requirements. With greater material width requirements and larger gore sections, providing defect free material sections will become more challenging. Quality control processes used by fabric manufacturers will be reviewed and discussed to ensure delivery of materials with consistency and minimal defects. Improved methods for inspection (mostly visual techniques) will need to be developed utilizing larger handling equipment and inspection tables. Very significant cost impacts can arise if defects are not discovered until late in the fabrication process. Improved quality control and inspection processes may not present difficult technical challenges, however they will require a significant near-term investment of resources to develop the necessary techniques and procedures.

\section{F-TPS Packing}

A $15 \mathrm{~m}$ HIAD system presents new challenges for packing operations that were not needed with the previous $6 \mathrm{~m}$ and $3 \mathrm{~m}$ HIADs. These smaller F-TPS articles were integrated with the IS and then folded and packed by hand into the proper shape. Final packing and compression was then performed using vacuum and a hydraulic ram to achieve the final stowage volume and pack density. The weight and bulk of a $15 \mathrm{~m}$ F-TPS cannot be manipulated by hand and will require custom equipment for lifting, folding, and pressing into the initial shape. Packing performed to reach the required density and shape will involve large equipment and high capacity hydraulic rams to provide the needed compression forces. A conceptual design for $15 \mathrm{~m}$ packing operations and requirements will be developed to guide support equipment design development and cost estimates.

In addition, the F-TPS design needs to consider the interface with the space vehicle, and performance. Features and construction methods used for large scale F-TPS must be tolerant of folding and high compression. The design of the F-TPS termination at the HIAD shoulder and the aft side attachment to the IS must be able to withstand the loads and strains due to packing. Another challenge for large scale articles will be stitching designs used for gore seaming and quilting between layers, which must be durable for packing to maintain performance after deployment. Packing survivability of F-TPS design features will initially be demonstrated and tested in small sample sections. After packing, F-TPS layer specimens will be thermally tested in arc jet or high temperature wind tunnel test facilities to verify performance. Full F-TPS articles will be integrated with the IS for packing and deployment cycle tests.

\section{F-TPS Load Testing}

Load testing of the F-TPS is required to demonstrate its capability to withstand the peak deceleration loads during atmospheric entry. There are no existing ground test facilities that can apply the required load and environment for a $15 \mathrm{~m}$ diameter HIAD. To date, the static load test fixture described in Section 3 and shown in Figure 9 has also been used for structural load testing of the F-TPS when integrated with the IS. This method was demonstrated with the previous $3-6 \mathrm{~m}$ HIAD test articles. This approach provides accurate load control and collection of corresponding shape and strain data. The F-TPS connections to the space vehicle and IS are demonstrated as well as the shape of the F-TPS surface since it is compressed against the IS. For a $15 \mathrm{~m}$-class HIAD, the challenge will be to identify an appropriate facility for the test fixture and associated costs. HIAD project development plans also propose subscale flight demonstrations from low Earth orbit to validate performance in relevant environments and entry conditions. These subscale tests will require significant investment in resources but are a necessary step for F-TPS development towards 15m-class HIADs intended for proposed Mars and Earth mission applications.

\section{HIAD AERoshell ScAling Benefits}

Contrary to the process of scaling high performance structures, there are noteworthy benefits of growing the HIAD aeroshell to a $15 \mathrm{~m}$-class system. Handmade textile structures can be afflicted by the resolution of human 
accuracy, and material component non-linearity. Improvements in both of these areas can be realized as the scale of components and magnitude of the design loads increase.

\section{Handmade Textile Accuracy}

Although the HIAD fabrication teams are a collection of expert tradesmen, there will always be a limitation in accuracy when manufacturing handmade textile structures. A reasonable accuracy at the high end of this field would be $1 / 8$ " when sizing and sewing materials. Within smaller structures, such as the $3 \mathrm{~m}$ IRVE-3 aeroshell, $1 / 8$ " of variability represented a $0.5 \%$ deviation in the nominal pairing strap length. This might seem small, but it can cause a large difference in load distribution between adjacent straps. The detrimental system level effects of this accuracy limitation were seen during the development of the IRVE-3 structure when the first few designs exhibited asymmetrical deflection under load due to uneven strap loading. Technologies were then developed to reduce the variability in the strap loads. Making the rational assumption that the manufacturing accuracy of $1 / 8$ " will remain constant when scaling up the HIAD structure, a resultant decrease in the strap length variation percentage will be seen. For instance, a scaled structure utilizing a 32 " minor diameter torus will only have about a $0.075 \%$ in pairing strap variation from the $1 / 8$ " accuracy as compared to the $0.5 \%$ from the $3 \mathrm{~m}$ HIAD. This indicates that it will be easier to have consistency in the hand fabricated structures as they increase in size, therefore creating better defined load paths and opportunities for greater efficiencies in the design.

\section{Material Response}

In addition to human accuracy, the response of the material components under load will improve as the aeroshell size increases. Many of the textile components currently used for the aeroshell design are greatly oversized in load capability because their designs were dictated by other variables. This forces the straps to operate in the non-linear region. As the aeroshell components grow in size and the design loads for the associated mission profiles increase, many of the same materials designs can be used. This means that these same material components would endure greater loads than they experienced on the smaller vehicles. Therefore, the textile elements will operate in the linear region of their structural response, making the load response of the system easier to analyze and resulting in a more efficient and consistent structure. This is a great opportunity for the HIAD team, but will require the development of an appropriate margin policy to maximize these benefits.

\section{CONCLUSION AND FUTURE WORK}

IS and F-TPS challenges associated with scaling up to a $15 \mathrm{~m}-$ class aeroshell, as well as others that have not been discussed, are to be addressed during the HIAD-2 ground test campaign. The HIAD project will continue to work closely with the contractor team members responsible for manufacturing these two systems to develop new and creative strategies to mitigate these challenges. In addition to addressing these scaling challenges, other development work will be conducted to increase the performance envelope of the HIAD system. This work will include developing asymmetric structures to generate lift and controllability, evolving new materials and additives to endure higher heating, advancing new structural designs to better distribute load, and maturing other enabling capabilities. These tasks and others will be addressed in a future publication.

Although scaling the current aeroshell design to a $15 \mathrm{~m}$-class system has many challenges, the HIAD team feels confident that the continued development of fruitful mitigation strategies with the guidance of experts on flexible systems will result in the successful fabrication and verification testing of a $12 \mathrm{~m}$ aeroshell by the end of the current ground test campaign.

\section{ACKNOWLEDGEMENTS}

The authors would like to thank NASA's Space Technology Mission Directorate, Game Changing Development Program, Langley Research Center, and Ames Research Center for their continued support to further the development of HIAD technology.

\section{REFERENCES}

[1] Smith, B.P. et al., "A Historical Review of Inflatable Aerodynamic Decelerator Technology Development," IEEE Aerospace Conference, IEEEAC paper\#1276, January 2010.

[2] Del Corso, J. A. et al., "Thermal Analysis and Testing of Candidate Materials for PAIDAE Inflatable Aeroshell' 20th AIAA Aerodynamic Decelerator Systems Conference, AIAA 2009-2925, May 2009.

[3] O'Keefe, S. A. et al, "IRVE-II Post-Flight Trajectory Reconstruction" AIAA Atmospheric Flight Mechanics Conference, AIAA 2010-7515, August 2010.

[4] Cassell, A. M., Swanson, G. T. et al., "Overview of the Hypersonic Inflatable Aerodynamic Decelerator Large Article Ground Test Campaign" 21 ${ }^{\text {st }}$ AIAA Aerodynamic Decelerator Systems Conference, AIAA 2011-2569, May 2011.

[5] Olds, A. D et al., "IRVE-3 Post-Flight Reconstruction" $22^{\text {nd }}$ AIAA Aerodynamic Decelerator Systems Conference, AIAA 2013-1390, March 2013.

[6] Johnson, Scott. "Forged in Fire, ULA's New Rocket, Vulcan, Revealed." RSS 20. Spaceflight Insider, 14 Apr. 2015. Web. 23 Oct. 2015.

\section{BIOGRAPHIES}

Neil Cheatwood has played key roles in NASA's planetary atmospheric flight programs. He is a nationally recognized expert in aerosciences and flight mechanics for planetary 
entry systems. He was the Principle Investigator for the Mars Entry, Descent, and Landing Instrumentation (MEDLI) project. He leads LaRC efforts to develop inflatable aeroshell technologies. He was the Principle Investigator for NASA LaRC's Inflatable Reentry Vehicle Experiment (IRVE), as well as the Program to Advance Inflatable Decelerators for Atmospheric Entry. Dr. Cheatwood was responsible for the entry aerodynamic databases for the Stardust, Mars Microprobe, Genesis, and Mars Exploration Rovers missions. He has also contributed to the Mars Global Surveyor and Mars Sample Return flight projects. Dr. Cheatwood is an AIAA Associate Fellow and the principle author or co-author of over 60 technical publications in the fields of fluid dynamics, atmospheric entry, and systems engineering.

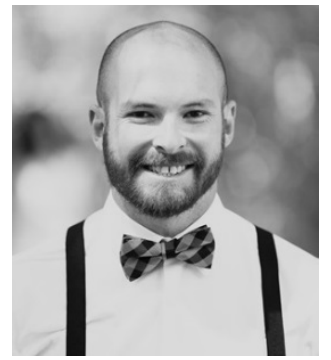

Greg Swanson is an AMA Inc. employee at NASA Ames Research Center in the Entry Systems and Vehicle Development Branch. His work involves a wide variety of component and system level test support including wind tunnel testing, arc jet testing, static load testing, and environmental testing. Mr. Swanson also creates custom sensor systems, wireless embedded sensing systems, and custom data acquisition system designs for a numerous number of technology development projects. Currently he mainly supports HIAD inflatable structure development and testing. Mr. Swanson received his BS and MS in Electrical Engineering from the University of Idaho in 2007 and 2009, respectively.

Keith Johnson is a senior aerospace engineer in the Atmospheric Flight and Entry Systems Branch at NASA LaRC. During his 25 years at LaRC, Mr. Johnson has performed design and analysis of mechanical and process systems, managed tests for complex mechanical and fluid systems, managed wind tunnel and test facility modification projects, served as systems engineer for flight projects, and served as COTR for several large projects. More recently, he has supported various space flight technology development projects including Mars Science Laboratory aeroshell design, Orion airbag landing system and Orion crew impact attenuation system. Over the last four years, he has led development of inflatable aeroshell structures for the HIAD project. Mr. Johnson has BS in Mechanical Engineering, Virginia Tech, 1984, and MS in Mechanical Engineering, Old Dominion University, 1995. 